and (6) and (7) together, in turn, imply $\int^{\infty}\left[\varphi_{i}(t)\right]^{2} d t<\infty$. Hence the functions

$$
\psi_{i}(t)=\left[\varphi_{i}(t)\right]^{-2} \int_{t}^{\infty}\left[\varphi_{i}(\tau)\right]^{2} d \tau
$$

exist for all $t \geqq t_{0}$ and are uniformly bounded; in fact, $a^{2} \leqq \psi_{i}(t) \leqq b^{2}$ where $a$ and $b$ are certain constants.

Now consider the function

$$
V(y, t)=\psi_{1}(t) y_{1}^{2}+\psi_{2}(t) y_{2}^{2}+\cdots+\psi_{n}(t) y_{n}^{2} .
$$

It evidently satisfies Liapounoff's criterion for asymptotic stability; it is a positive definite quadratic form, admitting of an infinitely small upper bound, and its derivative, by virtue of (2), becomes

$$
\frac{d V}{d t}=-\left(y_{1}^{2}+y_{2}^{2}+\cdots+y_{n}^{2}\right)+W(y, t)
$$

where $W(y, t)$ is a quadratic form whose coefficients depend upon those elements $b_{i j}(t)$ of $B(t)$ for which $i<j, i, j=1,2, \cdots n$. Since these elements can always be made sufficiently small by a transformation with constant coefficients (which will not affect stability properties) the derivative $d V / d t$ will be a negative definite quadratic form. Hence the trivial solution $y(t) \equiv 0$ of $(2)$ is asymptotically stable, and therefore the trivial solution $x(t) \equiv 0$ of $(1)$ is asymptotically stable. This establishes our theorem.

O. Perron* was the first to prove directly that the conditions

$$
\varphi_{i}(t) \leqq C_{1}, \quad \varphi_{i}(t) \int_{t_{0}}^{t} \frac{d \tau}{\varphi_{i}(\tau)} \leqq C_{2}
$$

are necessary and sufficient for the trivial solution $x(t) \equiv 0$ of (1) to be asymptotically stable.

*O. Perron, Die Stabilitaetsfrage bei Differentialgleichungen, Math. Zeitschrift 32, 703-728 (1930).

\title{
CONDITIONS SATISFIED BY THE EXPANSION AND VORTICITY OF A VISCOUS FLUID IN A FIXED CONTAINER*
}

By J. L. SYNGE (Dublin Institute for Advanced Studies)

1. Introduction. In plane motion of a viscous fluid inside a fixed container, the expansion $\theta$ and the vorticity $\omega$ cannot be arbitrarily assigned. A necessary and sufficient condition ${ }^{1}$ for the consistency of given $\theta$ and $\omega$ with vanishing velocity on the walls is

$$
\int(\theta U+2 \omega V) d S=0
$$

*Received April 17, 1951. This paper was written while the author was on leave of absence at the Institute for Fluid Dynamics and Applied Mathematics, University of Maryland.

1J. L. Synge, Quarterly of Applied Mathematics, 8, 107-108 (1950). The condition with $\theta=0$ was originally due to G. Hamel, Göttinger Nachr. Math.-Phys. Kl. 1911, 261-270. 
where $U, V$ is any pair of conjugate harmonic functions and the integration is taken over the region occupied by the fluid.

The purpose of the present paper is to extend this result to three dimensions. In space we have expansion $\theta$ and a vorticity vector $\omega_{i}$ (suffixes range $1,2,3$ with the summation convention), and the theorem which will be proved may be stated as follows: Given expansion $\theta$ and vorticity $\omega_{i}$ are consistent with vanishing velocity on the walls if, and only if,

$$
\int\left(\theta P+\omega_{i} Q_{i}\right) d V=0,
$$

where the integration is taken through the fluid, $Q_{i}$ being any (arbitrary) solution of the partial differential equations

$$
\Delta Q_{i}=Q_{k, k i}
$$

and $P$ satisfying

$$
P_{, i}=\frac{1}{2} \epsilon_{i j k} Q_{k, j} .
$$

Here $\epsilon_{i j k}$ is the usual permutation symbol, the comma denotes partial differentiation $\left(Y_{, i}=\partial Y / \partial x_{i}\right)$, and $\Delta$ the Laplace operator, so that $\Delta Q_{i}=Q_{i, k k}$. In vector notation (1.3) and (1.4) read $\nabla^{2} \mathbf{Q}=\nabla \nabla \cdot \mathbf{Q}$ and $\nabla P=\frac{1}{2} \nabla \times \mathbf{Q}$.

This theorem will be considered only for simply connected regions. For such regions, (1.3) are precisely the intcgrability conditions of (1.4), so that, given any solution of (1.3), $P$ exists satisfying (1.4), unique to within an additive constant.

2. Necessity of condition (1.2). Expansion $\theta$ and vorticity $\omega_{i}$ are connected with velocity $u_{i}$ by

$$
u_{i, i}=0, \quad \epsilon_{i j k} u_{k, i}=2 \omega_{i} .
$$

The problem of finding a motion with given $\theta$ and $\omega_{i}$ in a region $V$, bounded by a fixed surface $B$ to which the fluid adheres, is the problem of solving the partial differential equations (2.1) for $u_{i}$ with the boundary condition

$$
u_{i}=0 \quad \text { on } B .
$$

The theorem stated above asserts that (1.2) is a necessary and sufficient condition on $\theta$ and $\omega_{i}$ for the existence of this solution.

The necessity of the condition is easy to prove. We assume the existence of a solution of $(2.1)$ and (2.2).

By virtue of (2.1), it follows that for any $P$ and $Q_{i}$ at all (not subject to any conditions save those of smoothness) we have

$$
\begin{aligned}
\int\left(\theta P+\omega_{i} Q_{i}\right) d V+ & \int u_{i}\left(P_{, i}-\frac{1}{2} \epsilon_{i j k} Q_{k, i}\right) d V \\
& =\int\left[\left(u_{i} P\right)_{, i}+\omega_{i} Q_{i}-\left(\frac{1}{2} \epsilon_{i j k} u_{i} Q_{k}\right)_{, i}+\frac{1}{2} \epsilon_{i j k} u_{i, j} Q_{k}\right] d V \\
& =\int\left(n_{i} u_{i} P-\frac{1}{2} n_{j} \epsilon_{i j k} u_{i} Q_{k}\right) d B,
\end{aligned}
$$


the last integral being taken over the boundary $B$, on which $n_{i}$ is the unit normal, drawn outward.

Note that the above follows from (2.1) only. We now bring in (2.2). This makes the last integral in (2.3) vanish. If we then subject $P$ and $Q_{i}$ to (1.3) and (1.4), the second integral in the first line vanishes, and we are left with the equation (1.2); the necessity of (1.2) is thus established.

3. A lemma. The proof of sufficiency is harder. It rests on a lemma, for which the proof offered here is not mathematically rigorous, resting as it does on the assumption that a certain minimum is attained. A precise mathematical proof would of course have to specify the requisite smoothness of the bounding surface $B$ and of the tangential component assigned on it (see immediately below).

Lemma: Given the tangential component of a vector $Q_{i}$ on the boundary $B$ of a region $V$, then $Q_{i}$ exists satisfying (1.3) and this boundary condition.

To prove this (or at least make it plausible), consider the integral

$$
I(Q)=\int \epsilon_{i j k} Q_{k, j} \epsilon_{i r s} Q_{s, r} d V .
$$

In vector notation, the integrand is $(\nabla \times \mathbf{Q})^{2}$ and cannot be negative. Thus for all $Q_{i}$ satisfying the stated boundary condition, $I(Q)$ is bounded below. We assume that the minimum is actually attained by some vector field; let $Q_{i}$ be it.

Then, if $c$ is any constant, and $q_{i}$ any vector field with zero tangential component on $B$, it follows that

$$
I(Q) \leq I(Q+c q)
$$

and hence by the usual procedure associated with Dirichlet's principle,

$$
\int \epsilon_{i j k} Q_{k, j} \epsilon_{i r s} q_{s, r} d V=0
$$

for all such $q_{i}$. This may be transformed into

$$
\int \epsilon_{i j k} Q_{k, j} \epsilon_{i r s} q_{s} n_{r} d B-\int \epsilon_{i j k} Q_{k, j} \epsilon_{i r s} q_{s} d V=0 .
$$

But $\epsilon_{i r} q_{s} n_{r}$ is the tangential component of $q_{i}$, turned through a right angle, and so vanishes. Further

$$
\epsilon_{i j k} \epsilon_{i r s}=\delta_{j} \delta_{k s}-\delta_{j s} \delta_{k r}
$$

and so we get

$$
\int\left(\Delta Q_{k}-Q_{i, j k}\right) q_{k} d V=0 .
$$

Since $q_{i}$ is arbitrary except for the boundary condition it follows that the minimising $Q_{i}$ satisfies (1.3).

As pointed out already, $P$ then exists satisfying (1.4).

4. The sufficiency of (1.2). We assume that $\theta$ and $\omega_{i}$ satisfy (1.2) for all $Q_{i}$ and $P$ satisfying (1.3) and (1.4). We have to prove the existence of a solution $u_{i}$ of (2.1) and (2.2). 
Choose a particular solution of (1.3), (1.4): $P=1, Q_{i}=0$. Then (1.2) gives

$$
\int \theta d V=0 \text {. }
$$

Now choose $P=0, Q_{i}=W_{, i}$, the gradient of any scalar field $W$; these satisfy (1.3), (1.4) without restriction on $W$. Then (1.2) gives

$$
\int \omega_{i} W_{, i} d V=0
$$

or

$$
\int \omega_{i} n_{i} W d B-\int \omega_{i, i} W d V=0 .
$$

Hence, in view of the arbitrariness of $W$,

$$
\omega_{i, i}=0 \quad \text { in } V, \quad \omega_{i} n_{i}=0 \quad \text { on } B .
$$

We now try to solve (2.1) and (2.2). This we do in two steps, first solving the system

$$
u_{i, i}=\theta, \quad \epsilon_{i j k} u_{k, i}=2 \omega_{i},
$$

with the boundary condition

$$
u_{i} n_{i}=0 \quad \text { on } B .
$$

There is a well known procedure for this; we express $u_{i}$ in terms of a scalar potential and a vector potential, and obtain for these certain Poisson integrals, yielding particular solutions of (4.5). The problem of solving (4.5) and (4.6) is thus reduced to a Neumann problem, and it is known that (4.1) and (4.4) are sufficient conditions for the existence of a solution to (4.5) and (4.6).

The second step is to prove that the tangential component of this $u_{i}$ vanishes, the normal component being already zero by (4.6). This is easy. Equation (2.3) is valid, since it depends only on (2.1), i.e. (4.5). But the first two integrals in (2.3) vanish by (1.2) and (1.4) respectively. Also the first part of the last integrand vanishes by (4.6). So we are left with

$$
\int n_{j} \epsilon_{i j k} u_{i} Q_{k} d B=0 .
$$

But $\epsilon_{i j k} n_{j} Q_{k}$ is the tangential component of $Q_{i}$, turned through a right angle, and this, as we saw in the lemma, may be chosen arbitrarily. From this it follows at once that the tangential component of $u_{i}$ must vanish on $B$.

Thus the solution of (4.5) and (4.6) is in fact the solution of (2.1) and (2.2). This completes the proof of the sufficiency of the condition (1.2), subject of course to the assumption that the minimum of (3.1) is in fact attained.*

*Added in proof, June 22, 1951: In recent papers which the author had not seen when the present paper was written, C. Truesdell, Comptes Rendus Ac. Sci. Paris, 232, 1277, 1396 (1951), has given the condition (1.2) with consideration of its sufficiency, and this condition has also been given by $F$. $H$. van den Dungen, Q. Appl. Math. 9, 203 (1951), but the question of sufficiency has not been fully considered by him. 